\title{
Modeling Multirotor Aerodynamic Interactions Through the Vortex Particle Method
}

\author{
Eduardo Alvarez \\ Brigham Young University, ealvarez@byu.edu \\ Andrew Ning \\ Brigham Young University, aning@byu.edu
}

Follow this and additional works at: https://scholarsarchive.byu.edu/facpub

Part of the Engineering Commons

\section{Original Publication Citation}

Alvarez, E. J., and Ning, A., "Modeling Multirotor Aerodynamic Interactions Through the Vortex Particle Method," AIAA Aviation Forum, Dallas, TX, Jun. 2019. doi:10.2514/6.2019-2827

\section{BYU ScholarsArchive Citation}

Alvarez, Eduardo and Ning, Andrew, "Modeling Multirotor Aerodynamic Interactions Through the Vortex Particle Method" (2019). Faculty Publications. 3191.

https://scholarsarchive.byu.edu/facpub/3191

This Conference Paper is brought to you for free and open access by BYU ScholarsArchive. It has been accepted for inclusion in Faculty Publications by an authorized administrator of BYU ScholarsArchive. For more information, please contact ellen_amatangelo@byu.edu. 


\title{
Modeling Multirotor Aerodynamic Interactions Through the Vortex Particle Method
}

\author{
Eduardo J. Alvarez* and Andrew Ning ${ }^{\dagger}$ \\ Brigham Young University, Provo, Utah, 84602
}

\begin{abstract}
Distributed electric propulsion and vertical take-off and landing has recently opened a new design space for urban air mobility. However, the use of multiple rotors operating in close proximity introduces complicated aerodynamic interactions that are not well understood, are not captured through conventional design tools, and need to be addressed in the conceptual design stage. This study investigates the accuracy of the viscous vortex particle method (VPM) in modeling rotor-on-rotor aerodynamic interactions in a sideby-side configuration as encountered in tilt-rotor, quadrotor, and distributed propulsion aircraft. The VPM approach has the potential to enable the use of mid/high fidelity models capturing multirotor interactions during conceptual design. Validation of the individual rotor is presented in both hovering and forward-flight configurations at both low and high Reynolds numbers. Validation of the hovering multirotor is then presented, followed by a detailed parametric study of rotor-to-rotor interactions during hover and forward flight, constructing the response surface of thrust, torque, and propulsive efficiency as a function of operational parameters.
\end{abstract}

\section{Introduction}

Technology convergence in the past ten years has recently opened a new design space in electric aircraft, enabling the use of distributed propulsion and electrical vertical take-off and landing (eVTOL) for urban air mobility. ${ }^{1-4}$ The concept of eVTOL in an urban setting is forecasted to evolve into a $\$ 1.5$ trillion "flying car" industry by the year 2040. ${ }^{5}$ However, this unconventional aircraft configuration poses technical challenges that still remain to be solved. ${ }^{6}$ A strong noise signature ${ }^{7,8}$ and a complicated transition maneuver ${ }^{9,10}$ are examples of the challenges encountered in eVTOL aircraft, both stemming in some degree from the aerodynamic interactions between rotors and lifting surfaces. ${ }^{11}$ As an example of eVTOL configuration, Fig. 1 shows NASA's GL-10 tilt-rotor prototype aircraft in hovering mode during take off, prior to transitioning into wing-borne forward flight.

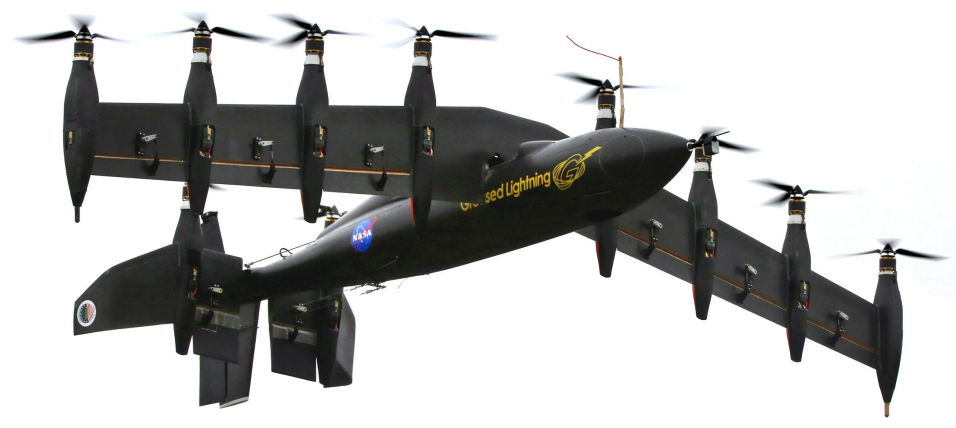

Figure 1: NASA GL-10 eVTOL prototype aircraft in hover mode during take off. Credits: NASA Langley/David C. Bowman.

*Doctoral Candidate, Department of Mechanical Engineering, ealvarez@byu.edu, AIAA Member.

$\dagger$ Assistant Professor, Department of Mechanical Engineering, aning@byu.edu, AIAA Senior Member. 
The use of multiple rotors operating in close proximity introduces complicated aerodynamic interactions that are not well understood, are not captured through conventional design tools, and need to be addressed in the conceptual design stage. ${ }^{12-14}$ For instance, Yoon et al. ${ }^{15}$ investigated the effects of separation distance between rotors on a quad tilt-rotor aircraft in hover, discovering a myriad of beneficial and counterproductive interactions between rotors and airframe. Using an unsteady detached-eddy Reynolds-average Navier-Stokes (RANS) solver, they simulated the rotors operating at a high Reynolds number with and without the aircraft body. As they varied the separation between rotors, they observed a drop in thrust as low as $4 \%$ at a separation $10 \%$ of the rotor diameter, and observed an ingestion of recirculating vorticity from above the rotors into the wake caused by rotor interactions. When the fuselage and wings were included in the simulation, the rotors were benefited from body-on-rotor interactions increasing the thrust by about $3 \%$. However, both wing and fuselage create a large download caused by the rotor downwash impinging on them, resulting in a net loss of vertical force of about $16 \%$ due to the accumulation of rotor-on-rotor, rotor-on-body, and body-on-rotor interactions. These complicated dynamics between rotors and the aircraft body illustrate the need to better understand the aerodynamic interactions incountered in eVTOL aircraft and address them in the early stages of design.

Coaxial and tandem rotor interactions have been studied for decades in the rotorcraft community, meanwhile the side-by-side multirotor configuration has remained relatively unexplored. Recent experimental works ${ }^{16-20}$ have studied the counter-rotating configuration where neighboring rotors spin in opposing directions (clockwise versus counterclockwise), however, no attention has been given to the co-rotating configuration where neighboring rotors spin in the same direction (both clockwise, or both counterclockwise). Also, these studies only considered the hovering case, meanwhile the effects of multirotor interactions in forward flight remain unexplored. Numerous studies of quadrotor and helicopter forward flight are found in the literature, but forward flight in a distributed propulsion eVTOL configuration differs from a quadrotor or helicopter configuration. In the latter, the flight velocity produces an edgewise or tilted flow since the rotors provide both the lift and thrust of the vehicle. In contrast, distributed propulsion eVTOL aircraft are typically wing-borne during cruise, and the axes of rotation of the rotors are roughly aligned with the direction of flight, with the velocity of the aircraft adding directly to the axial inflow and advance ratio of the rotors. Even though previous studies have shown that multirotor interactions could drop the thrust performance by only a small amount ${ }^{15-17,20}(\sim 4 \%$ relative to the isolated rotor), they also show that noise could be increased by as much as $3 \mathrm{~dB},{ }^{18,19}$ corresponding to an acoustic signature two times more intense, which is a critical factor to consider in the design of urban mobility aircraft. Experimental results indicate that this increase in noise is directly related to unsteady loading during aerodynamic interactions. Hence, accurately modeling these aerodynamics interactions will lay the groundwork for future aeroacoustic modeling and noise reduction during the conceptual design stage.

In this study we will investigate the accuracy of the viscous Vortex Particle Method ${ }^{22,23}$ (VPM) in modeling rotor-on-rotor aerodynamic interactions in a side-by-side configuration as encountered in tilt-rotor, quadrotor, and distributed propulsion aircraft. The viscous VPM is a meshless method for solving the vorticity form of Navier-Stokes equations in a Lagrangian scheme. The VPM is a direct numerical simulation of vorticity-governed flows that accurately preserves vortical structures and is absent of the numerical dissipation associated to conventional mesh-based approaches like RANS and large-eddy simulation (LES).
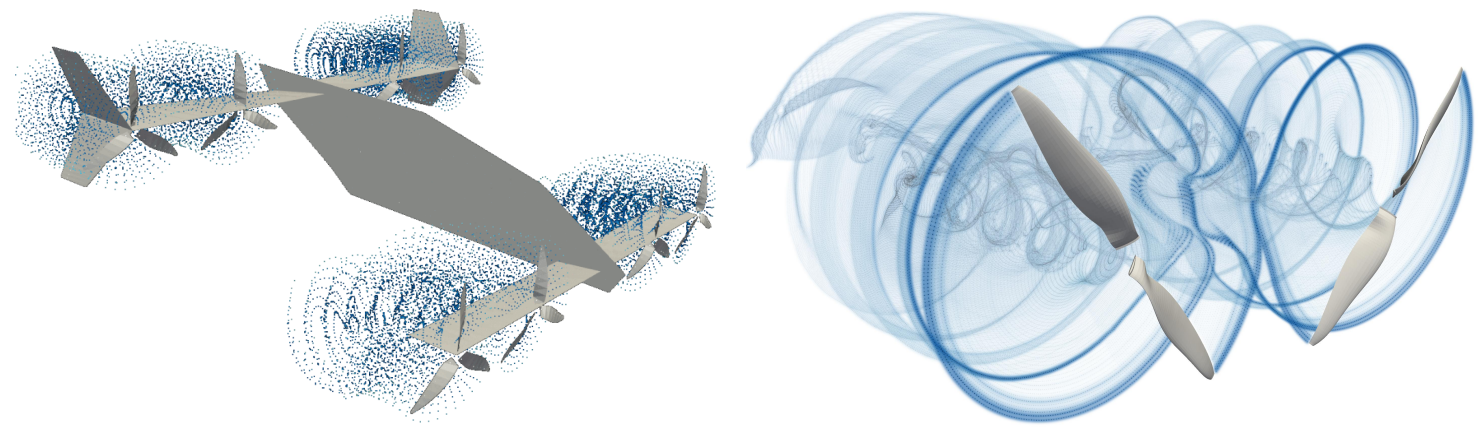

Figure 2: Low-fidelity VPM simulation of eVTOL aircraft (left) and high-fidelity VPM simulation of multirotor wake mixing ${ }^{21}$ (right). 
This method has been shown to achieve a continuum of fidelity levels, from low fidelity apt for conceptualdesign ${ }^{24-31}$ to high fidelity apt for research-quality computational fluid dynamics, ${ }^{32-35}$ while offering a considerable speed up over a RANS or LES approach. Fig. 2 shows a low-fidelity simulation of a full-aircraft configuration and a high-fidelity simulation of wake mixing of two counter-rotating rotors from previous work by the authors using VPM. ${ }^{21}$ In recent work, VPM has been coupled with a noise code based on the Ffowcs Williams-Hawkings equation, successfully predicting rotor acoustics associated to unsteady loading. ${ }^{36}$ Furthermore, the method has been shown to be CPU and GPU parallelizable, and scalable in heterogeneous systems for high performance computing. ${ }^{37-39}$ Thus, the VPM approach has the potential to enable the use of mid/high-fidelity aeroacoustic models capturing multirotor interactions during conceptual design.

In Section II, the theory of the VPM will be summarized and the propeller model will be presented. In Section III, validation of the individual rotor will be presented in both hovering and forward-flight configurations at both low and high Reynolds numbers. Finally, validation of the hovering multirotor will be presented in Section IV, followed by a detailed parametric study of rotor-to-rotor interactions during hover and forward flight, constructing response surfaces of thrust, torque, and propulsive efficiency as a function of operational parameters. The parameters will be tip-to-tip separation distance $s$, Reynolds number $\operatorname{Re}_{\mathrm{D}}$ (associated with the RPM), advance ratio $J$ (associated with the flight speed), and direction of rotation (counter or co-rotation). The response surfaces will be constructed from mid-fidelity VPM simulations sweeping in all four operational parameters, resulting in a total of 1152 simulations.

\section{Modeling Methodology}

In this section, the theory of the viscous vortex particle method is summarized detailing the implementation used in this study (Section II.A). A propeller model based in VPM and blade elements is presented in Section II.B, and the test configurations along with respective rotor geometries are described in Section II.C.

\section{II.A. Viscous Vortex Particle Method}

\section{II.A.1. Governing Equations}

A viscous, incompressible flow dominated by vorticity can be resolved by formulating the Navier-Stokes equations into their vorticity form. This is done by taking the curl over the original momentum equation

$$
\nabla \times\left(\frac{\partial \mathbf{u}}{\partial t}+(\mathbf{u} \cdot \nabla) \mathbf{u}\right)=\nabla \times\left(-\frac{1}{\rho} \nabla p+\nu \nabla^{2} \mathbf{u}\right)
$$

leading to an expression that is not dependent on the pressure field:

$$
\frac{D \boldsymbol{\omega}}{D t}=(\boldsymbol{\omega} \cdot \nabla) \mathbf{u}+\nu \nabla^{2} \boldsymbol{\omega} .
$$

The material derivative expressed in Eq. (1), and the material-conservative nature of the vorticity makes the $\boldsymbol{\omega}$ field especially fit for a Lagrangian description. In order to do so, the field is discretized into vortex particles $^{22}$ as a radial basis function interpolation of basis $\zeta_{\sigma}$ and coefficients $\boldsymbol{\Gamma}_{p}$ :

$$
\boldsymbol{\omega}(\mathbf{x}, t) \approx \sum_{p} \boldsymbol{\Gamma}_{p}(t) \zeta_{\sigma}\left(\mathbf{x}-\mathbf{x}_{p}(t)\right)
$$

Each particle represents a volume of fluid that travels with the local velocity as in Eq. (3), where $\mathbf{x}_{p}$ is the position of the $p$-th particle. The coefficient $\boldsymbol{\Gamma}_{p}$, termed vectorial circulation or vectorial vortex strength, is defined as $\boldsymbol{\Gamma}_{p} \equiv \int_{\operatorname{vol}_{p}} \boldsymbol{\omega} \mathrm{d} V$ and approximated as $\boldsymbol{\Gamma}_{p} \approx \boldsymbol{\omega}_{p} \operatorname{vol}_{p}$, where $\boldsymbol{\omega}_{p}$ is the vorticity associated with the $p$-th particle of volume $\operatorname{vol}_{p}$. Applying this particle discretization to Eq. (1), it is obtained that the vectorial circulation of each particle evolves as in Eq. (4), where the first right-hand-side term is the vortex stretching, while the second represents a scheme for modeling the viscous diffusion $\nu \nabla^{2} \boldsymbol{\omega}$.

$$
\begin{aligned}
\frac{\mathrm{d}}{\mathrm{d} t} \mathbf{x}_{p}(t) & =\mathbf{u}\left(\mathbf{x}_{p}(t), t\right) \\
\frac{\mathrm{d}}{\mathrm{d} t} \boldsymbol{\Gamma}_{p}(t) & =\left(\boldsymbol{\Gamma}_{p}(t) \cdot \nabla\right) \mathbf{u}\left(\mathbf{x}_{p}(t), t\right)+\left.\frac{\mathrm{d}}{\mathrm{d} t} \boldsymbol{\Gamma}_{p}(t)\right|_{\text {visc }}
\end{aligned}
$$




\section{II.A.2. Velocity Kernel}

By the Helmholtz Decomposition Theorem, the incompressible velocity field $\mathbf{u}$ dominated by vorticity is defined by some vector potential field $\boldsymbol{\psi}$ as $\mathbf{u}(\mathbf{x}, t)=\nabla \times \boldsymbol{\psi}(\mathbf{x}, t)$. Then, from the definition of the vorticity field $(\boldsymbol{\omega}=\nabla \times \mathbf{u})$, we get the three-dimensional unbounded Poisson's problem

$$
\nabla^{2} \boldsymbol{\psi}=-\boldsymbol{\omega}
$$

which can also be rewritten using the particle approximation from Eq. (2) as

$$
\nabla^{2} \boldsymbol{\psi}(\mathbf{x}) \approx-\sum_{p} \boldsymbol{\Gamma}_{p} \zeta_{\sigma}\left(\mathbf{x}-\mathbf{x}_{p}\right)
$$

The Poisson's problem yields that the velocity induced by the field of vortex particles is calculated as

$$
\mathbf{u}(\mathbf{x})=\sum_{p} g_{\sigma}\left(\mathbf{x}-\mathbf{x}_{p}\right) \mathbf{K}\left(\mathbf{x}-\mathbf{x}_{p}\right) \times \mathbf{\Gamma}_{p},
$$

where $g_{\sigma}$ is a normalized smoothing function calculated from the chosen basis function $\zeta_{\sigma}$, and $\mathbf{K}(\mathbf{x})=$ $-\frac{\mathbf{x}}{4 \pi\|\mathbf{x}\|^{3}}$ is the singular Newtonian kernel resulting from the three-dimensional Green's function of the unbounded Poisson's problem. In this study we implement the Gaussian basis function

$$
\zeta_{\sigma}(\mathbf{x})=\frac{1}{\left(2 \pi \sigma^{2}\right)^{3 / 2}} \exp \left(-\frac{\|\mathbf{x}\|^{2}}{2 \sigma^{2}}\right) .
$$

\section{II.A.3. Fast Multipole Method}

The governing equations, Eqs. (3) and (4), coupled with the velocity kernel Eq. (5) require the calculation of all particle interactions with a computational complexity of order $\mathcal{O}\left(N^{2}\right)$, where $N$ is the number of particles. This complexity is prohibitive since the number of particles readily escalates to the order of the hundreds of thousands in mid-fidelity simulations. In order to ease the computation in this study, the fast multipole method (FMM) is used for the computation of the governing equations. The FMM approximates pairwise interactions of particles through spherical harmonic functions, ${ }^{40,41}$ reducing the original computational complexity to a problem of order $\mathcal{O}(N)$. The computation of the vortex stretching is performed through an efficient complex-step derivative approximation as explained in previous work, ${ }^{21}$ implemented in a modified version of the open-source, parallelized FMM code ExaFMM. ${ }^{42,43}$

\section{II.A.4. Viscous Diffusion}

In this study, the viscous diffusion term in Eq. (4) is solved through the core spreading method ${ }^{44}$ coupled with the radial basis function (RBF) interpolation approach developed by Barba, ${ }^{23,45-48}$ which avoids the need for particle splitting while allowing the viscous VPM to be a truly meshless method. In the core spreading method, a Gaussian radial basis allows the viscous diffusion term in Eq. (1) to be solved by spreading the smoothing radius as

$$
\frac{\mathrm{d} \sigma^{2}}{\mathrm{~d} t}=2 \nu
$$

however, this approach is a convergent approximation to the Navier-Stokes equations only for small core sizes. $^{44}$ This introduces the need to resize all cores to their initial size after they grow larger than an arbitrary threshold. A popular solution to this problem is the splitting of overgrown particles, but this increases the number of particles exponentially in time. Barba introduced an alternative method for core resizing by resetting all core sizes $\sigma$ to an initial value after a certain time, and estimating the new vectorial circulations $\boldsymbol{\Gamma}_{p}$ that preserves the same vorticity field $\boldsymbol{\omega}$ through an RBF interpolation. This method not only takes care of the core-overgrowth problem, but also allows for spatial adaptation, and the RBF can be efficiently implemented in the FMM evaluation through the generalized minimal residual (GMRES) method. In our implementation, we have assumed a uniform core size throughout the particle field, allowing us to solve the RBF through the conjugate gradient method. 


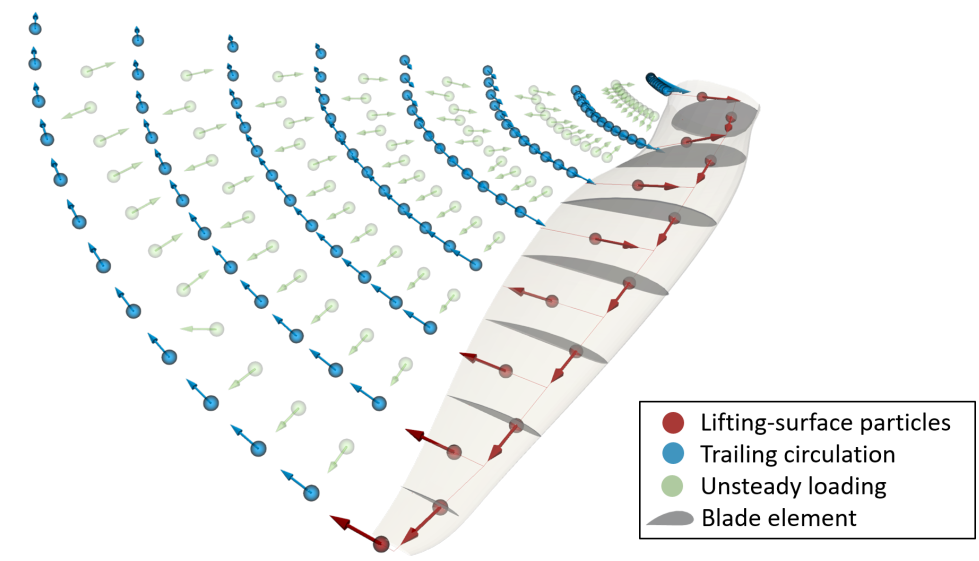

Figure 3: Blade and wake deployment in the propeller model. Particles are colored by their respective source of vorticity; arrows indicate the direction of vortex strength $\boldsymbol{\Gamma}_{p}$.

\section{II.B. Propeller Model}

In this study, each blade of the propeller is modeled as a rotary lifting surface where the physics of interest have been broken down into three aspects: load distribution, blade-induced velocity, and wake-induced velocity. The load distribution is calculated through two-dimensional blade elements, and is used for deriving the circulation along the lifting surface. In turn, the blade-induced velocity is obtained from the circulation distribution by embedding vortex particles along the surface that preserve such circulation, as shown in Fig. 3. As the blade moves, the trailing vorticity is shed off the trailing edge as free-particles from both the unsteady loading and shed circulation. Thus, blade and wake-induced velocities are all computed through the FMM, allowing for efficient scaling of the fidelity of the simulation to the order of millions of particles, and all unsteady dynamics are resolved as the VPM steps in time. Fig. 4 shows the computation flow diagram of the simulation.

In the simulation setup step (blue block in Fig. 4), the user specifies the rotor geometry and blades are discretized into elements. Two-dimensional aerodynamic airfoil characteristics of each blade element are precomputed through XFOIL at the corresponding local Reynolds number, and a Prandtl-Glauert compressibility correction is applied to the lift curve, thus capturing both viscosity and compressibility effects. Both lift and drag curves are adjusted to capture three-dimensional drag and stall-delay effects ${ }^{49}$ encountered in rotor blades, and the Viterna method ${ }^{50}$ is applied to obtain post-stall $\pm 180^{\circ}$ extrapolations of these curves.

In the first time step of the simulation, the inflow at every blade element is calculated from freestream and rotational velocity and used for calculating the effective angle of attack (AOA) of the element. From the precalculated airfoil lift and drag curves, the effective angle of attack is used to look up the lift and drag at every element, and the load distribution of every blade is thus determined. In all subsequent time steps, the propellers are rotated and vortex particles are shed off trailing edges. The Navier-Stokes equations are solved in their vorticity form (Eq. (1)) calculating the convection, stretching, and viscous diffusion of the vortex particles (Eqs. (3) and (4)). The velocity induced by the wake and lifting surfaces is then added to the freestream and rotational velocities in order to calculate the new effective angle of attack, and the load distribution is updated (gray block in Fig. 4). The process is then iterated until the end of the simulation, $t_{f}$, as shown in the green block. This algorithm is similar to the method developed by Jo and Lee, ${ }^{28,31}$ except that we omit the minor iterations and let load distributions convergence in time as the wake is deployed. Also, we model the lifting surfaces through embedded vortex particles in order to perform all computation through the VPM, which allows us to scale the computation efficiently in order $\mathcal{O}(N)$ through the FMM.

In a previous study ${ }^{21}$ we have shown that the VPM is able to fully characterize the evolution of the wake, from near field to far field and transition into turbulent breakdown. Furthermore, the VPM is a direct-numerical simulation approach, requiring an increased spatial resolution in the turbulent breakdown regime to ensure convergence. In order to ease computation in this study, the wake will be partially removed once in the breakdown regime as described in Alvarez and Ning. ${ }^{21}$ 


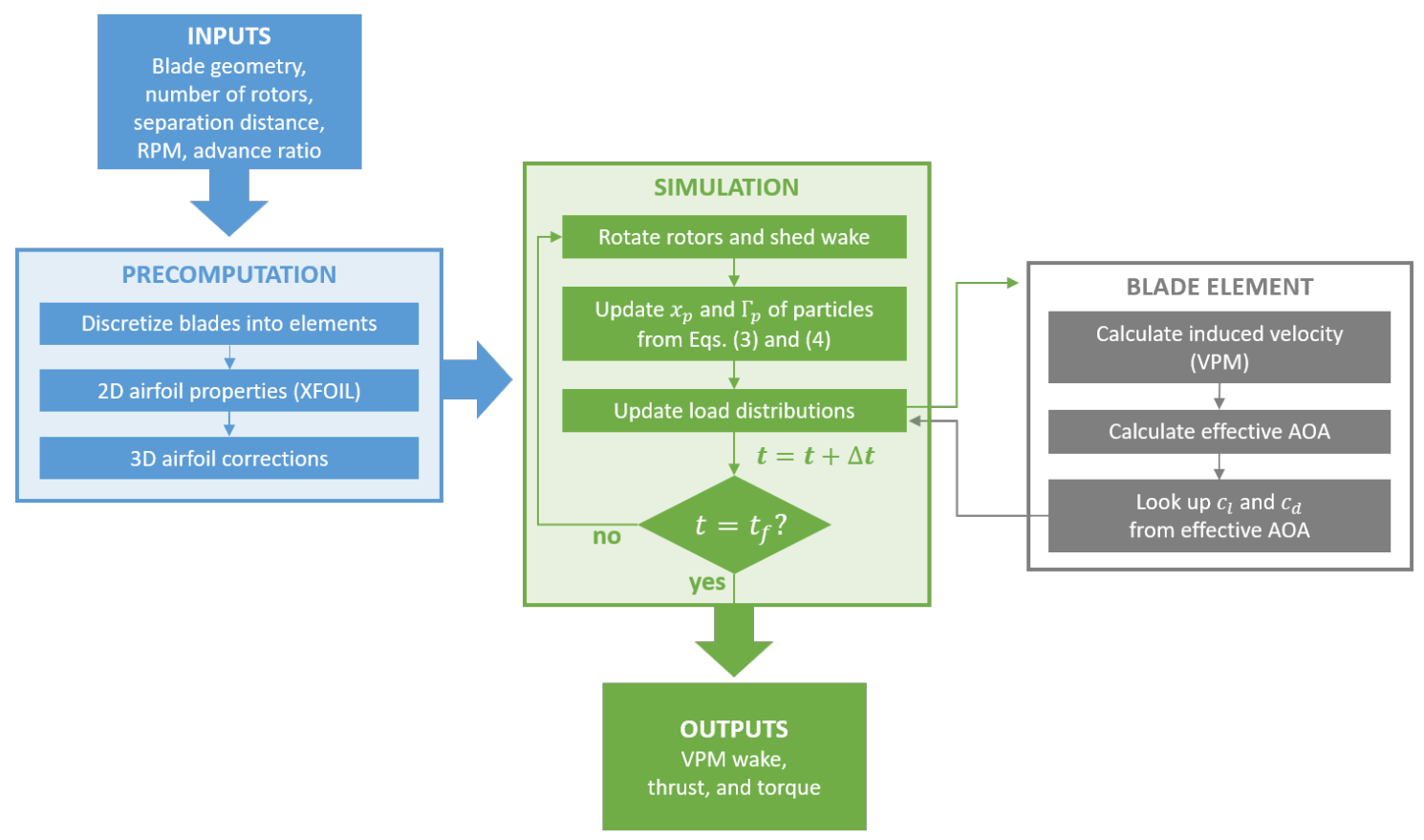

Figure 4: Computation flow diagram of the propeller model.

\section{II.C. Test Cases}

In order to simulate both hovering and forward-flight multirotor configurations at both low and high Reynolds numbers, two different rotor geometries are used. The hovering case uses a quadcopter rotor matching the geometry of the DJI Phantom II rotor described by Zhou et al. ${ }^{18}$ and Ning, ${ }^{19}$ and the low-Reynolds simulation of side-by-side hovering rotors is compared to reported particle image velocimetry (PIV) and load cell measurements. This rotor is $240 \mathrm{~mm}$ in diameter, and its geometry approximates the optimum rotor design obtained by imposing a constant effective angle of attack along the blade. The geometry was digitized assuming the twist distribution $\theta(r)=5.3^{\circ}+6.3^{\circ} \frac{1}{r}$ (with $r$ the non-dimensional radial position) and an uniform E63 airfoil shape transitioning to an E856 airfoil towards the hub. The chord and leading edge distributions are generated as described by Ning. ${ }^{19}$ All simulations are run at $4860 \mathrm{RPM}$ and no freestream velocity, matching the experimental configuration resulting in a diameter-based Reynolds number $\operatorname{Re}_{D}$ of $6.5 \times 10^{5}$ and chord-based Reynolds of $\operatorname{Re}_{c}$ of $6.2 \times 10^{4} \cdot \operatorname{Re}_{D}$ and $\operatorname{Re}_{c}$ are calculated at the $70 \%$ blade span as

$$
\operatorname{Re}_{D}=\frac{V_{70 \%} D}{\nu} \text { and } \operatorname{Re}_{c}=\frac{V_{70 \%} \bar{c}}{\nu}
$$

where $\bar{c}$ is the mean chord, and $V_{70 \%}$ is the effective speed resulting from both freestream and local rotational velocity at $70 \%$ the blade span.

The forward-flight configuration uses the APC thin-electric 10x7 rotor operating at a high Reynolds number. This rotor is a hobby-grade model commonly used on small unmanned aircraft, it is readily available, and its 10-inch diameter makes it a good fit for mid-size wind tunnel testing. Moreover, its performance has been measured and indexed in the UIUC Propeller Database, ${ }^{51}$ and verified by McCrink and Gregory ${ }^{52}$ both experimentally and through blade-element momentum theory. The blade is generated assuming an uniform NACA 4412 airfoil shape, using the chord and twist distributions digitized by McCrink and Gregory. Note that forward flight in distributed-propulsion aircraft implies a fully axial inflow, instead of the edgewise flow encountered in rotorcraft. The performance of the individual rotor is validated against experimental data across a sweep of advance ratio $J$ at a constant $\operatorname{Re}_{D}$ of $1.5 \times 10^{6}$ and $\operatorname{Re}_{c}$ of $1.2 \times 10^{5}$, where $J$ is calculated as $J=\frac{V_{\infty}}{n D}$, with $n=\frac{\mathrm{RPM}}{60}$.

The thrust coefficient $C_{T}$, torque coefficient $C_{Q}$, and propulsive efficiency $\eta$ hereby reported are defined 


$$
C_{T}=\frac{T}{\rho n^{2} D^{4}}, \quad C_{Q}=\frac{Q}{\rho n^{2} D^{5}}, \quad \text { and } \quad \eta=\frac{T V_{\infty}}{2 \pi n Q} .
$$

In all validation cases, temporal integration is performed in a third-order Runge-Kutta scheme at a time step equivalent to a $5^{\circ}$ blade rotation; blades are discretized into 50 elements; and spatial resolution of the wake is dictated by imposing a smoothing radius twice the distance between contiguous particles shed off the blade tip, shedding two particles at every time step. Particles are shed all along the blade trailing edge.

\section{Single-rotor Validation}

Prior to multirotor modeling, the propeller model is first validated in a single-rotor configuration at both low and high Reynolds number. In this section the single rotor is simulated in both hover and forward flight, and results are compared to experimental measurements reported by Zhou et al.. ${ }^{18}$ Ning, ${ }^{19}$ and McCrink and Gregory. ${ }^{52}$

\section{III.A. Hover Case}

The hover configuration uses the DJI Phantom II rotor operating at a low Reynolds number $\left(\operatorname{Re}_{c}=6.2 \times 10^{4}\right)$ and constant $4860 \mathrm{RPM}$. The thrust coefficient $C_{T}$ of the rotor throughtout the simulation is shown in Fig. 5 along with the experimental mean thrust and fluctuation. The wake starts to develop and convect downstream during the first two iterations, and the near field becomes fully developed after only three iterations as indicated by the convergence of thrust. The periodic spikes observed at six, ten, and fourteen revolutions correspond to every instance when the turbulent breakdown region has been trimmed. The wake after eighteen revolutions is shown in Fig. 6 (left), where features of the topology can be clearly identified: a well-defined structure in the near field $(z<0.5 D)$, an onset of leapfrogging at $z \approx 0.5 D$, and a transition into turbulent breakdown at $z \approx 1.0 D$. The simulation converges to a mean $C_{T}$ of 0.1013 and a steady fluctuation (standard deviation) of 0.001 , meanwhile Zhou et al. ${ }^{18}$ and Ning ${ }^{19}$ report an experimental mean $C_{T}$ of 0.1007 and a fluctuation of 0.008 . This results in an error of only $0.5 \%$ between the simulation and experimental mean $C_{T}$, meanwhile the fluctuation is largely underpredicted. We conjecture that the fluctuations observed in the experimental measurements of the individual rotor are caused by interactions with the test stand not captured in our simulations (e.g., mounting pole, hub, and mechanical vibrations).

Fig. 7 compares the ensemble-average velocity in the single rotor (left figures) observed experimentally and predicted at a plane located a distance $0.1 D$ downstream. The simulation shows a streamtube slightly more contracted than what is observed experimentally. Fig. 8 shows the in-plane vorticity of both the experimental PIV and the simulated single rotor (left figures). The vorticity distribution is obtained at a

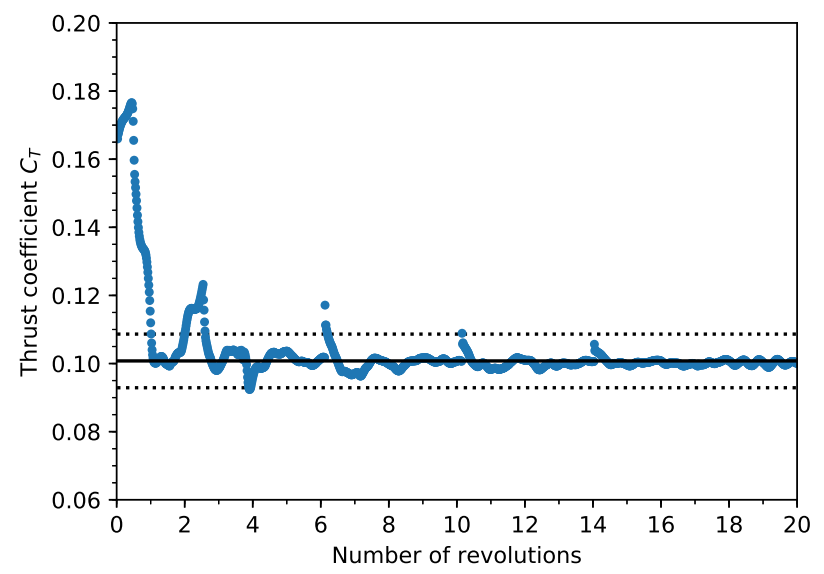

Figure 5: Convergence of thrust coefficient $C_{T}$ (blue dots) in hovering single-rotor simulation. Solid black line corresponds to the experimental mean thrust and dashed lines enclose the standard deviation as reported in Zhou et al. ${ }^{18}$ and Ning. ${ }^{19}$ 


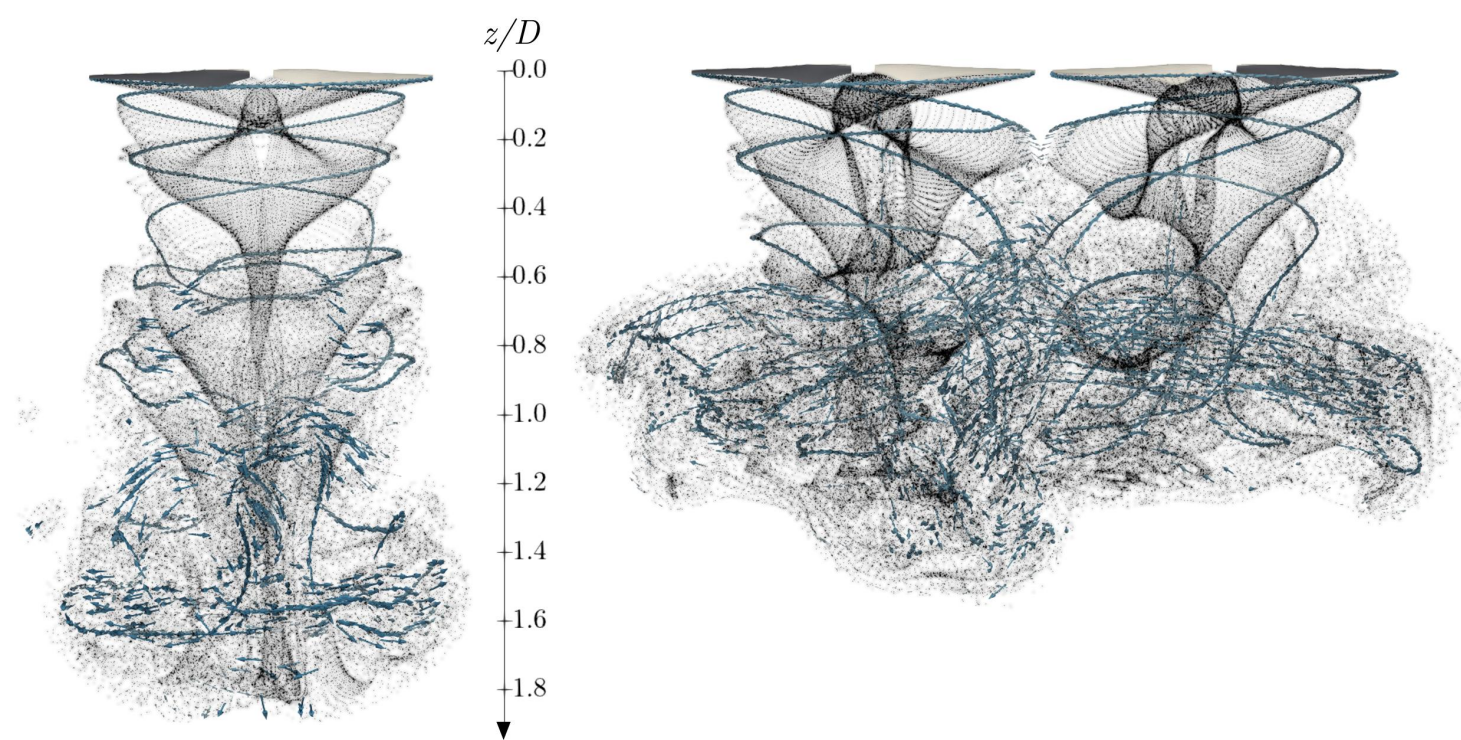

Figure 6: Wake of single-rotor and multirotor hover simulations. Arrows scaled by the vortex strength of every particle.

phase-locked angle of $120^{\circ}$, where the phase is defined as the angle between the vertical $y z$-plane and the position of the rotor blade. It can be seen that the VPM simulation correctly predicts an onset of tip-vortex leapfrogging at a downstream distance of about $z=0.5 \mathrm{D}$. Thus, the propeller model seems to captures all the physics of interest at low Reynolds number in a hovering single rotor configuration.

\section{III.B. Forward-flight Case}

The forward-flight configuration uses the APC 10x7 rotor operating at a high Reynolds number (constant $\operatorname{Re}_{c}$ of $1.2 \times 10^{5}$ ) across a sweep of advance ratio $J$. Fig. 9 compares the predicted thrust, torque, and propulsive efficiency to the experimental values reported by McCrink and Gregory. ${ }^{52}$ It is observed that both $C_{T}$ and $C_{Q}$ match the experimental values at low and moderate advance ratios, meanwhile they start to drop earlier than what is seen experimentally at high advance ratios. This is also observed in the blade-element momentum predictions by McCrink and Gregory, which hints that this may be an issue with the geometry description (i.e., inaccurate airfoil shape or twist distribution differing from the actual rotor) rather than the modeling method. The discrepancies in $C_{T}$ and $C_{Q}$ cancel out as the propulsive efficiency $\eta$ is calculated, resulting in a good agreement across all advance ratios up to $J \approx 0.725$. Thus, the VPM propeller model seems to be valid at high Reynolds number across low and moderately-high advance ratios in forward-flight single-rotor configuration.

\section{Multirotor Results}

In this section, the hovering multirotor case is compared to the experimental measurements reported by Zhou et al. ${ }^{18}$ and Ning, ${ }^{19}$ validating the VPM multirotor model. Finally, a parametric study of rotor-on-rotor interactions across all advance ratios and Reynolds numbers is presented.

\section{IV.A. Hover Case}

The hovering single-rotor case of Section III.A is now extended to multirotor configuration by adding an identical rotor in counter-rotation at a tip-to-tip distance $s$ of $0.05 D$. Counter-rotation means that one propeller rotates clockwise, meanwhile the other rotates counter-clockwise. The simulation shows that the multirotor wake mixing onsets a topological instability that moves the turbulent-breakdown region closer to the plane of rotation, as shown in Fig. 6 (right). Fig. 8 compares the in-plane vorticity field between single-rotor and multirotor configuration. Both simulation and experiment show that tip vortices lose their 

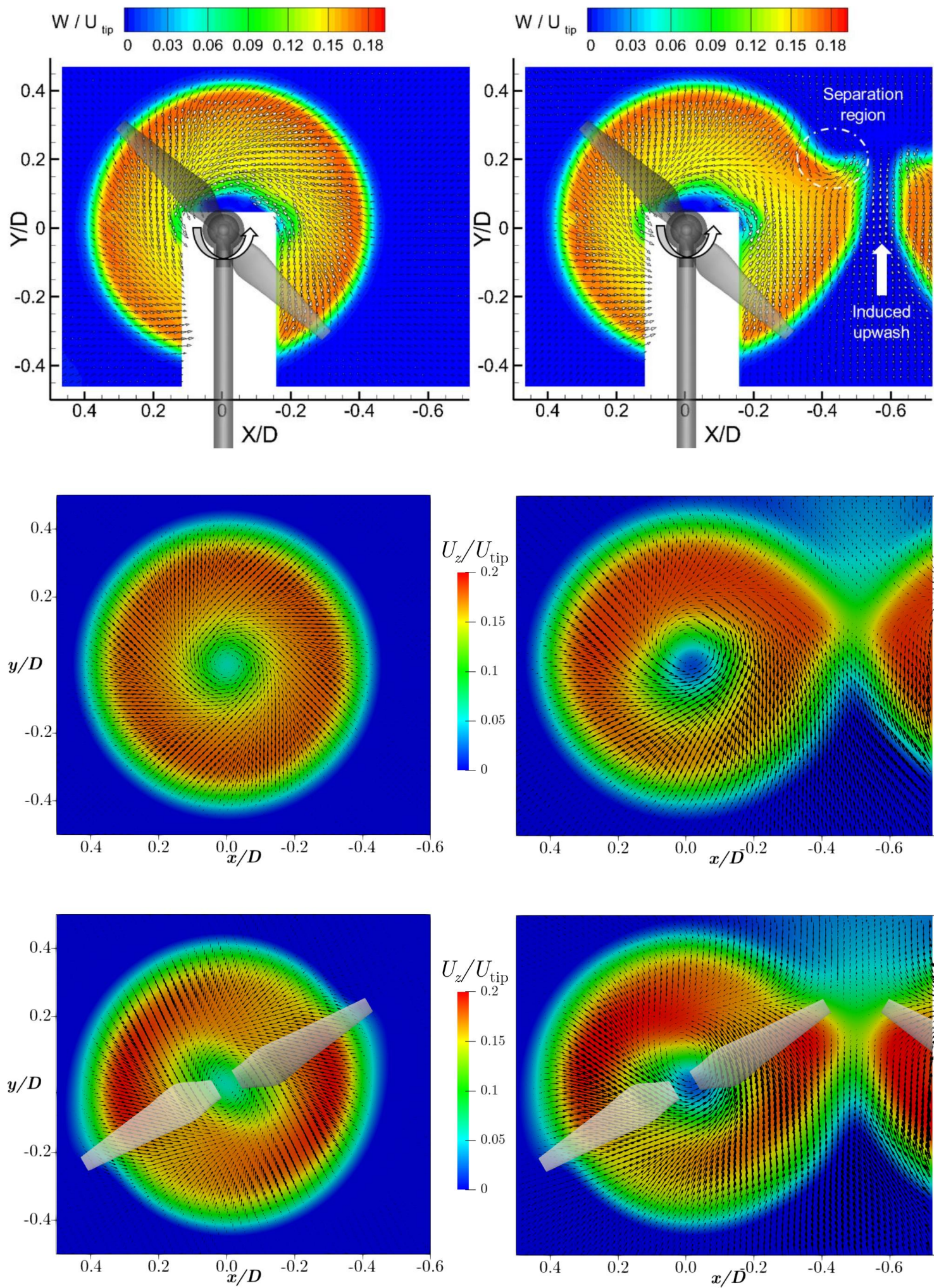

Figure 7: Velocity field at plane $z=0.1 D$ of single rotor (left) and multirotor (right, $s=0.05 D$ ) in hover configuration. Top row shows the experimental ensemble average (retrieved from Zhou et al. ${ }^{18}$ ), middle row shows the simulation ensemble average, and bottom row shows the simulation phase-locked field at $120^{\circ}$ (see blade positions). Colormap corresponds to axial velocity, while arrows indicate swirl velocity. 

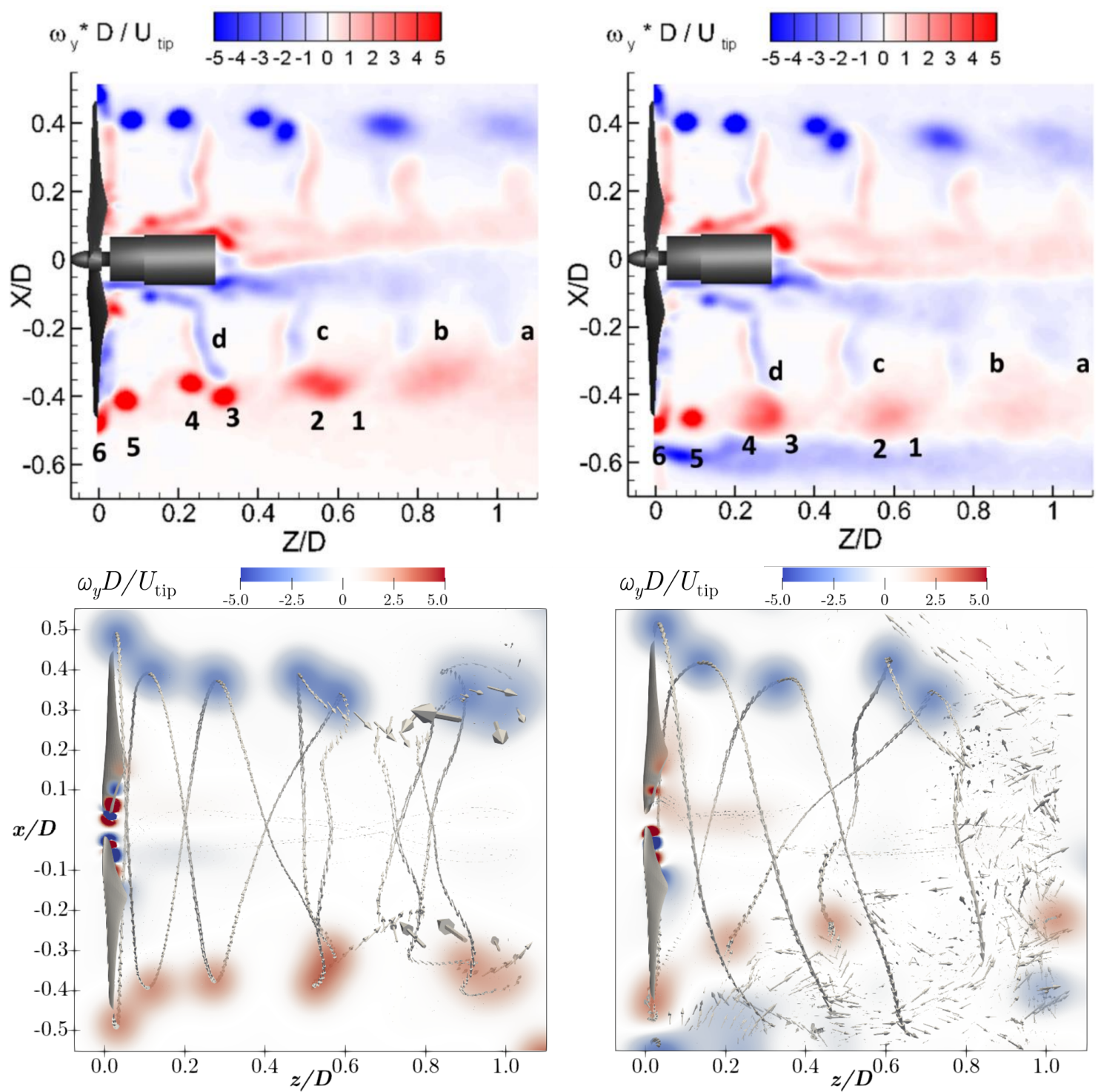

Figure 8: Phase-locked vorticity distribution of single rotor (left) and multirotor (right, $s=0.05 D$ ) in hover configuration as measured experimentally (top) and simulated (bottom). Arrows scaled by the vortex strength of every particle. Experimental measurements retrieved from Zhou et al. ${ }^{18}$
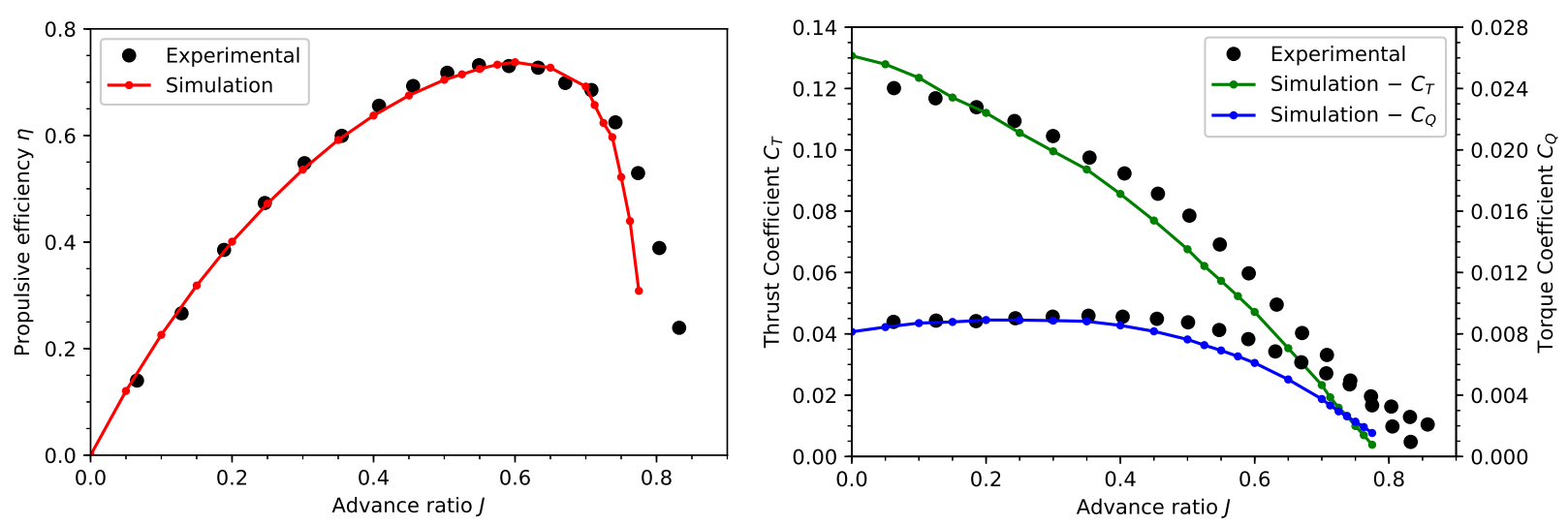

Figure 9: Comparison between simulation and experimental thrust, torque, and propulsive efficiency in forward-flight single-rotor configuration. 
structure as wakes mix in-between the rotors $(x=-0.5 D$ region, compare left and right figures).

Next, the separation distance $s$ was varied to determine its effects on thrust. Fig. 10 shows the mean thrust and fluctuations compared to what Zhou et al. ${ }^{18}$ observed experimentally, normalized by the values obtained in the single-rotor configuration. Every time that blades pass through the region of wake mixing, the loading of the blade drops, decreasing the mean thrust and increasing the fluctuation, which is accentuated as the tip-to-tip distance becomes small. It is especially important that this unsteady loading is accurately captured since the noise augmentation observed experimentally stems from these fluctuations. Here we see that the VPM propeller model is able to capture these rotor-on-rotor interactions with reasonable accuracy ${ }^{\mathrm{a}}$, making it a good candidate for a future application in noise prediction.

Zhou et al. observed an accentuated upwash and a recirculation region in the near field between the rotors (shown in Fig. 7, top right), and conjectured that the flow separates along the blades every time they pass through this region, leading to the observed drop in blade loading. The simulation (Fig. 7, middle right) shows the same accentuated upwash observed experimentally, however the recirculation region is absent. The phase-locked field (Fig. 7, bottom right) shows a more accurate topology, yet no recirculation is seen. Since the drop in thrust shown in Fig. 10 agrees well between simulation and experiment in spite of not capturing such recirculation region, we conclude that the effects of the recirculation pocket are negligible and that the induced upwash is the main contributor to the drop in blade loading.

\section{IV.B. Hover and Forward-flight Parametric Study}

Previous studies on side-by-side multirotor interactions available in the literature have only explored the hovering configuration since fully characterizing the response surface beyond a hovering configuration poses a great challenge due to its multidimensionality. Leveraging the computational speed of the VPM, we have swept across all ranges of advance ratio $J$, Reynolds number $\operatorname{Re}_{D}$, separation distance $s$, and direction of rotation (counter and co-rotation). This resulted in a total of 1152 simulations ${ }^{\mathrm{b}}$ requiring a wall-clock time of 4 days in a desktop computer Intel(R) Xeon(R) CPU E5-2699 v3 @ 2.30GHz.

Fig. 11 shows the response surface of propulsive efficiency $\eta$ and thrust fluctuation (standard deviation of $C_{T}$ ) in counter-rotating APC 10x7 rotors at low, moderate, and high Reynolds numbers. Efficiency values have been normalized by the corresponding value obtained in single-rotor configuration. The solid surfaces correspond to radial basis function interpolations fitted to data points, whereas each data point corresponds to one VPM simulation. In the efficiency response surface (left figures), it is observed that multirotor interactions are detrimental across all advance ratios and Reynolds numbers, dropping the performance as
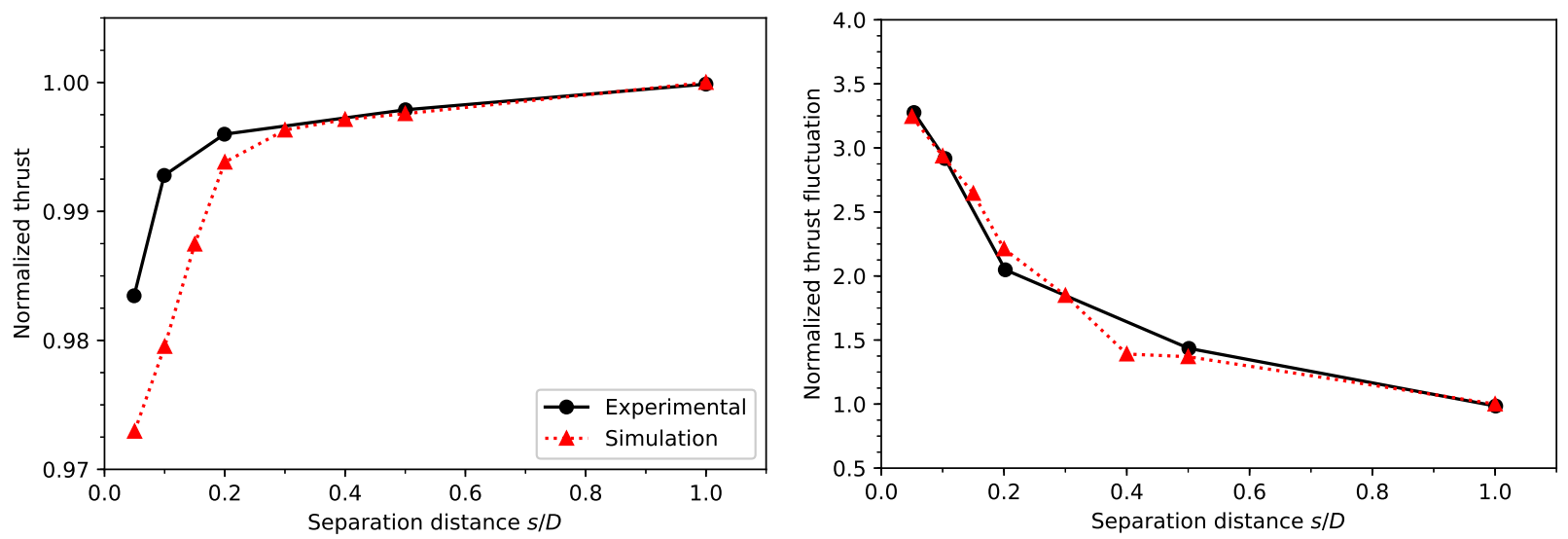

Figure 10: Effects of rotor-on-rotor interactions on thrust as the separation between rotors is decreased, normalized by their respective values in the single-rotor configuration.

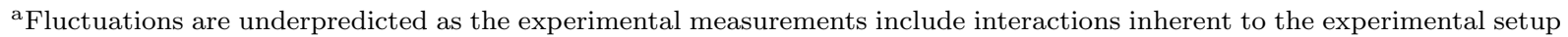
(mounting pole and hub) not captured in the simulation, as mentioned in Section III.A. However, simulation and experiment show good agreement after normalizing both by their respective single-rotor values.

b These are mid-fidelity simulations with 25 elements per blade and 18 time steps per revolution, shedding four particles per step with a smoothing core overlap twice the distance between contiguous particles, simulating a total of six revolutions in moderate/high advance-ratio cases, and 13 revolutions in low advance ratio cases $(J \leq 0.2)$. 

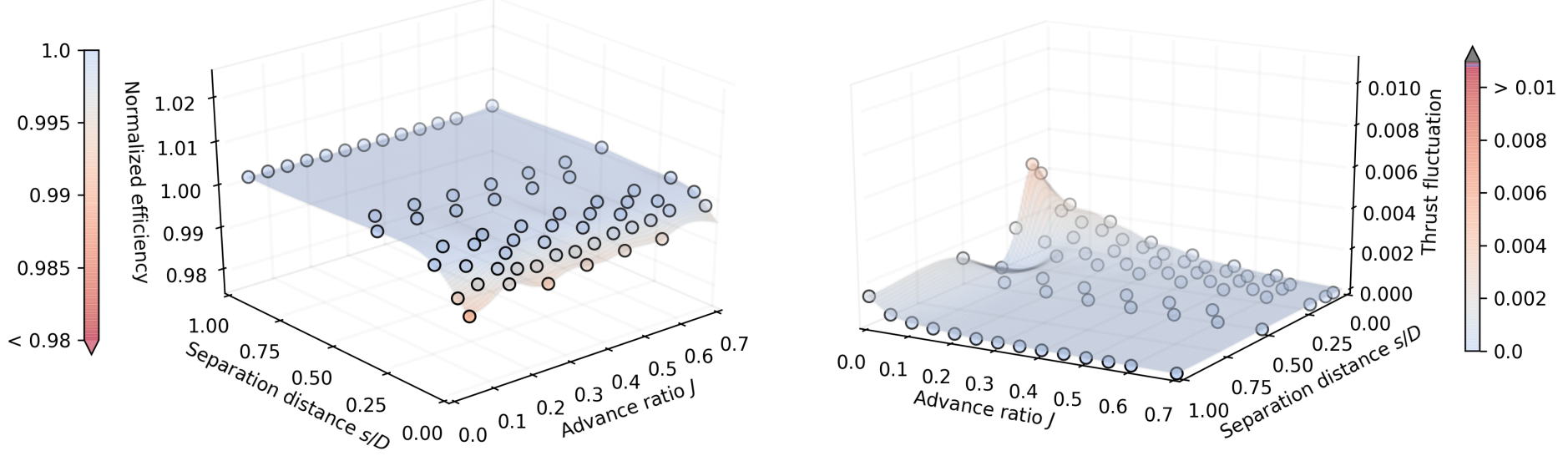

(a) Low Reynolds number $\left(\operatorname{Re}_{D}=1 \times 10^{5}\right)$
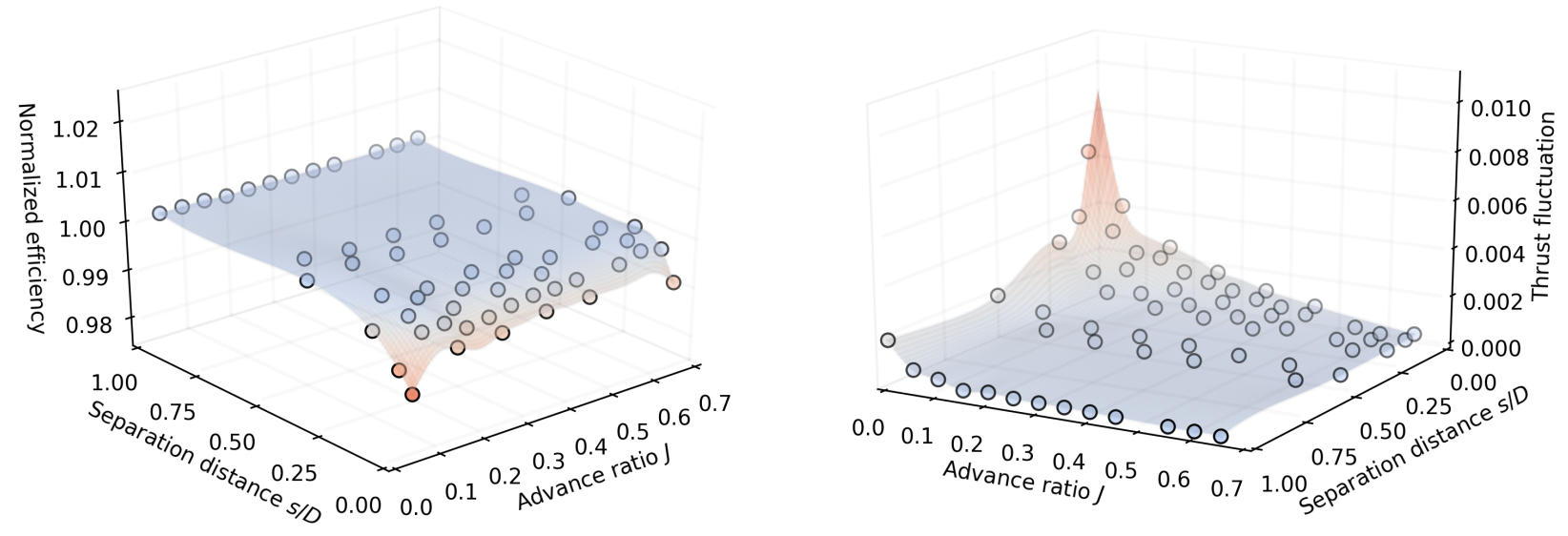

(b) Moderate-low Reynolds number $\left(\operatorname{Re}_{D}=5 \times 10^{5}\right)$
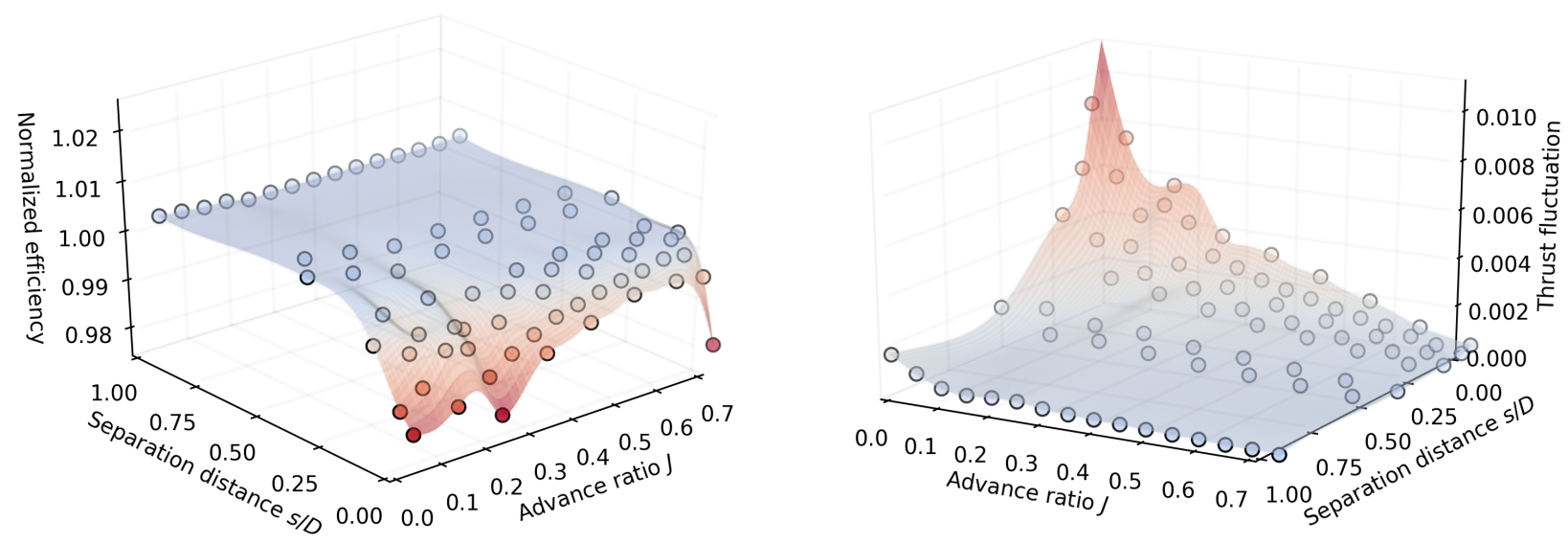

(c) High Reynolds number $\left(\operatorname{Re}_{D}=1.5 \times 10^{6}\right)$

Figure 11: Response surfaces of propulsive efficiency (normalized by corresponding single-rotor value) and thrust fluctuation (standard deviation) in counter-rotating APC 10x7 multirotor. Solid surfaces correspond to radial basis function interpolations fitted to data points (circles). Each data point corresponds to one VPM simulation. 
the distance between rotors become small. Furthermore, this drop in performance becomes more accentuated at low advance ratios. This is better observed in Fig. 12, which shows slices of the response surface at multiple Reynolds numbers at $s=0.05 D$, which evidences a trend of decreasing performance as the advance ratio decreases. Another observation from Figs. 11 and 12 is that a higher Reynolds number accentuates the negative interactions. The opposite was observed in an experimental study by Shukla et al., ${ }^{16,17}$ which may indicate that this trend on Reynolds number is dependent on the specific rotor design being studied (the APC 10x7 is designed for high-Reynolds operation with a loading distribution gradually decreasing towards the blade tip, whereas Shukla et al. used an untapered, untwisted blade highly loaded at the tip, designed for low-Reynolds testing and strong tip vortices).

The response surface of thrust fluctuation (Fig. 11, right figures) shows that fluctuations are accentuated at hover and near-hover configurations $(J<0.2)$ across all Reynolds numbers . This means that hover and near-hover can be expected to be the configurations of greatest noise associated to unsteady loading. It is also observed that Reynolds number has the effect of increasing thrust fluctuations accordingly. This is explained by the fact that a higher Reynolds number correlates to a higher RPM, which leads to an increased blade loading and stronger blade-tip vortices that accentuate rotor-on-rotor interactions in the APC 10x7. All trends already mentioned are consistent between counter and co-rotating configurations. Finally, Fig. 13 compares the effects of rotation direction (counter or co-rotation) on propulsive efficiency across Reynolds numbers and advance ratios at $s=0.05 D$, showing that a counter-rotating configuration (e.g., one propeller rotating clockwise while the other counter-clockwise) has a slight penalty over co-rotation at moderate and high advance ratios $(J>0.25)$ that is accentuated at a high Reynolds number, droping the efficiency as much as $0.5 \%$ lower than the co-rotating configuration at $\operatorname{Re}_{D}=1.5 \times 10^{6}$.

\section{Conclusion}

In this study we have shown the capability of the viscous vortex particle method to capture multirotor aerodynamic interactions in both hover and forward-flight configuration as encountered in distributed propulsion eVTOL aircraft at both low and high Reynolds numbers. Single-rotor validation has been presented, showing an error of only $0.5 \%$ between experimental and predicted thrust coefficient in hover, and good agreement on predicted propulsive efficiency across all advance ratios in forward flight configuration. Wake dynamics in multirotor hovering configuration showed a good qualitative agreement between simulation and particle-image velocimetry measurements. The simulation indicates that the accentuated induced upwash in between the rotors is the main contributor to the drop in blade loading during rotor-on-rotor interaction. The multirotor VPM model was shown to capture the thrust drop observed experimentally as the tip-to-tip distance between rotors became small, along with the associated thrust fluctuation.

A parametric study of rotor-on-rotor interactions during hover and forward flight in the APC 10x7 rotor

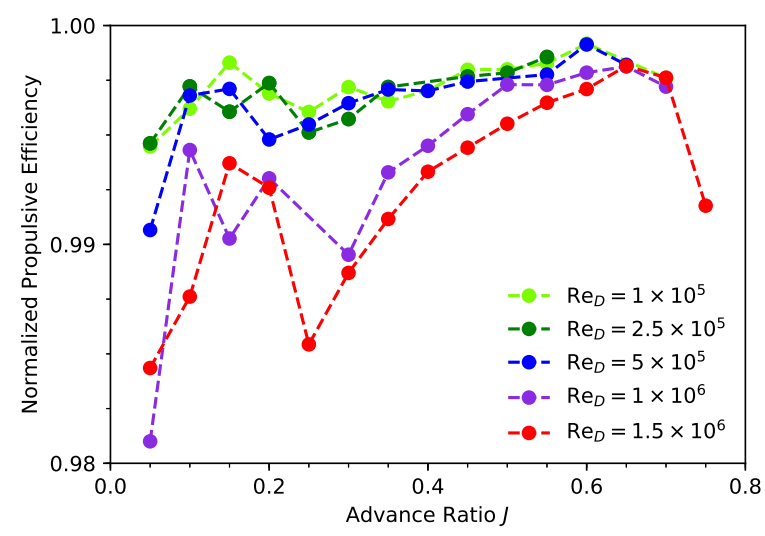

Figure 12: Slices of the efficiency response surface at different Reynolds numbers at $s=0.05 D$ on counter-rotating APC 10x7 multirotor.

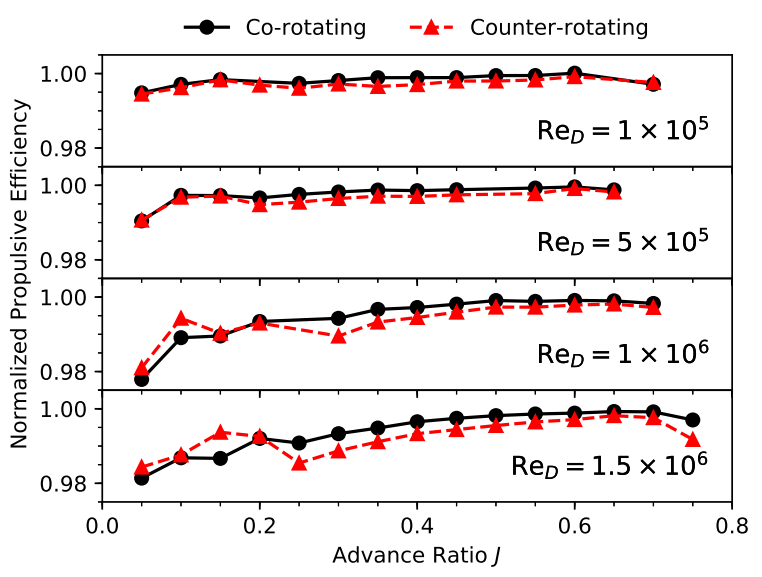

Figure 13: Effects of rotation direction on propulsive efficiency across advance ratios and Reynold numbers on APC 10x7 multirotor at $s=0.05 D$. 
revealed the following:

- Multirotor interactions are detrimental across all advance ratios and Reynolds numbers, dropping the propulsive efficiency as the distance between rotors becomes small.

- The performance drop becomes accentuated at low advance ratios.

- The largest thrust fluctuations (and potentially highest noise signature) are encountered in hover and near-hover configurations.

- At moderate and high advance ratios $(J>0.25)$, a counter-rotating configuration is slightly less efficient than a co-rotating one.

- A higher Reynolds number accentuates the interactions encountered in a rotor with lightly loaded blade tips, as is the APC $10 x 7$.

These tentative conclusions require further verification with higher-fidelity simulations reducing numerical uncertainty along with more exhaustive experimental validation, which will be performed in future work.

The aerodynamic validation of the VPM multirotor model hereby presented lays the groundwork for the future development of an aeroacoustic method for predicting the noise signature associated to unsteady loading in multirotor configurations, with the potential of integrating these analyses in the conceptual design stage of eVTOL aircraft.

\section{Acknowledgments}

This work is funded by the High Impact Doctoral Research fellowship granted by Brigham Young University.

\section{References}

${ }^{1}$ Silva, C., Johnson, W. R., Antcliff, K. R., and Patterson, M. D., "VTOL Urban Air Mobility Concept Vehicles for Technology Development," 2018 Aviation Technology, Integration, and Operations Conference, 2018, pp. 1-16.

${ }^{2}$ Moore, M., "NASA Puffin Electric Tailsitter VTOL Concept," 10th AIAA Aviation Technology, Integration, and Operations (ATIO) Conference, American Institute of Aeronautics and Astronautics, Reston, Virigina, sep 2010, pp. 1-12.

${ }^{3}$ Johnson, W., Silva, C., and Solis, E., "Concept Vehicles for VTOL Air Taxi Operations," AHS Technical Conference on Aeromechanics Design for Transformative Vertical Flight, 2018.

${ }^{4}$ Kasliwal, A., Furbush, N. J., Gawron, J. H., McBride, J. R., Wallington, T. J., De Kleine, R. D., Kim, H. C., and Keoleian, G. A., "Role of flying cars in sustainable mobility," Nature Communications, Vol. 10, No. 1, dec 2019 , pp. 1555.

5 "Flying Cars: Investment Implications of Autonomous Urban Air Mobility," Tech. rep., Morgan Stanley Research, 2018.

${ }^{6}$ Brelje, B. J. and Martins, J. R., "Electric, hybrid, and turboelectric fixed-wing aircraft: A review of concepts, models, and design approaches," Progress in Aerospace Sciences, , No. June, 2018, pp. 1-19.

${ }^{7}$ Pascioni, K. A., Rizzi, S. A., and Schiller, N., "Noise Reduction Potential of Phase Control for Distributed Propulsion Vehicles," AIAA Scitech 2019 Forum, No. January, American Institute of Aeronautics and Astronautics, Reston, Virginia, jan 2019, pp. 1-16.

${ }^{8}$ Ingraham, D., Gray, J. S., and Lopes, L. V., "Gradient-Based Propeller Optimization with Acoustic Constraints," AIAA Scitech 2019 Forum, No. January, American Institute of Aeronautics and Astronautics, Reston, Virginia, jan 2019 , pp. 1-12.

${ }^{9}$ Persson, L. and Lawrence, B., "Design and Control of an Experimental Tiltwing Aircraft," Tech. rep., NASA, 2017.

${ }^{10}$ Droandi, G., Syal, M., and Bower, G., "Tiltwing Multi-Rotor Aerodynamic Modeling in Hover, Transition and Cruise Flight Conditions," AHS International 74th Annual Forum 85 Technology Display, 2018, pp. 2018.

${ }^{11}$ Droandi, G., Gibertini, G., Grassi, D., Campanardi, G., and Liprino, C., "Proprotorwing aerodynamic interaction in the first stages of conversion from helicopter to aeroplane mode," Aerospace Science and Technology, Vol. 58, nov 2016, pp. 116-133.

${ }^{12}$ Borer, N. K., Derlaga, J. M., Deere, K. A., Carter, M. B., Viken, S., Patterson, M. D., Litherland, B., and Stoll, A., "Comparison of Aero-Propulsive Performance Predictions for Distributed Propulsion Configurations," 55th AIAA Aerospace Sciences Meeting, 2017, pp. 1-16.

${ }^{13}$ Theodore, C. R., "A Summary of the NASA Design Environment for Novel Vertical Lift Vehicles (DELIVER) Project," AHS Paper 20180001325, 2018.

${ }^{14}$ Ventura Diaz, P. and Yoon, S., "High-Fidelity Computational Aerodynamics of Multi-Rotor Unmanned Aerial Vehicles," 2018 AIAA Aerospace Sciences Meeting, 2018, pp. 1-22.

${ }^{15}$ Yoon, S., Lee, H. C., and Pulliam, T. H., "Computational Analysis of Multi-Rotor Flows," 54th AIAA Aerospace Sciences Meeting, , No. January, 2016, pp. 1-11.

${ }^{16}$ Shukla, D., Hiremath, N., and Komerath, N. M., "Low Reynolds Number Aerodynamics Study on Coaxial and QuadRotor," 2018 Applied Aerodynamics Conference, 2018. 
${ }^{17}$ Shukla, D. and Komerath, N., "Multirotor Drone Aerodynamic Interaction Investigation," Drones, Vol. 2, No. 4, 2018, pp. 43.

${ }^{18}$ Zhou, W., Ning, Z., Li, H., and Hu, H., "An Experimental Investigation on Rotor-to-Rotor Interactions of Small UAV Propellers," 35th AIAA Applied Aerodynamics Conference, , No. June, 2017, pp. 1-16.

${ }^{19}$ Ning, Z., Experimental investigations on the aerodynamic and aeroacoustic characteristics of small UAS propellers, Ph.D. thesis, Iowa State University, Digital Repository, Ames, 2018.

${ }^{20}$ Shukla, D. and Komerath, N., "Low Reynolds number multirotor aerodynamic wake interactions," Experiments in Fluids, Vol. 60, No. 4, apr 2019, pp. 77.

${ }^{21}$ Alvarez, E. J. and Ning, A., "Development of a Vortex Particle Code for the Modeling of Wake Interaction in Distributed Propulsion," 2018 Applied Aerodynamics Conference, American Institute of Aeronautics and Astronautics, jun 2018, pp. 1-22.

${ }^{22}$ Winckelmans, G. and Leonard, A., "Contributions to Vortex Particle Methods for the Computation of Three-Dimensional Incompressible Unsteady Flows," Journal of Computational Physics, Vol. 109, No. 2, dec 1993, pp. $247-273$.

${ }^{23}$ Barba, L. A., Leonard, A., and Allen, C. B., "Advances in viscous vortex methods - Meshless spatial adaption based on radial basis function interpolation," International Journal for Numerical Methods in Fluids, Vol. 47, No. 5, 2005 , pp. 387-421.

${ }^{24}$ Willis, D. J., Peraire, J., and White, J. K., "A combined pFFT-multipole tree code , unsteady panel method with vortex particle wakes," , No. October, 2005, pp. 1-6.

${ }^{25}$ Calabretta, J. S. and Mcdonald, R. A., "A Three Dimensional Vortex Particle-Panel Method for Modeling PropulsionAirframe Interaction," 48th AIAA Aerospace Sciences Meeting Including the New Horizons Forum and Aerospace Exposition, , No. January, 2010, pp. 2010.

${ }^{26}$ Berdowski, T. J., 3D Lagrangian VPM-FMM for Modelling the Near-Wake of a HAWT, Thesis, Technical University of Denmark and Delft University of Technology, 2015.

${ }^{27}$ Singh, P. and Friedmann, P. P., "Application of Vortex Methods to Coaxial Rotor Wake and Load Calculations in Hover," Journal of Aircraft, Vol. 55, No. 1, 2017, pp. 1-9.

${ }^{28}$ Jo, Y., Lee, H., and Lee, D. J., "Prediction of rotor flow for unmanned aerial system using nonlinear vortex lattice method," 6th Asian-Australian Rotorcraft Forum and Heli Japan 2017, ARF 2017, 2017.

${ }^{29}$ Teixeira, P. and Cesnik, C. E., "Propeller Effects on the Dynamic Response of HALE Aircraft," 2018 AIAA/ASCE/AHS/ASC Structures, Structural Dynamics, and Materials Conference, American Institute of Aeronautics and Astronautics, Reston, Virginia, jan 2018, pp. 1-15.

${ }^{30}$ Ho, J. C. and Yeo, H., "Assessing Calculated Blade Loads of the Tilt Rotor Aeroacoustic Model," Journal of Aircraft, Vol. 55, No. 3, may 2018, pp. 1287-1298.

${ }^{31}$ Lee, H. and Lee, D. J., "Numerical investigation of the aerodynamics and wake structures of horizontal axis wind turbines by using nonlinear vortex lattice method," Renewable Energy, Vol. 132, 2019, pp. 1121-1133.

${ }^{32}$ Stock, M., Gharakhani, A., and Stone, C., "Modeling Rotor Wakes with a Hybrid OVERFLOW-Vortex Method on a GPU Cluster," 28th AIAA Applied Aerodynamics Conference, , No. July, 2010, pp. 1-12.

${ }^{33}$ Chatelain, P., Duponcheel, M., Caprace, D.-G., Marichal, Y., and Winckelmans, G., "Vortex particle-mesh simulations of vertical axis wind turbine flows: from the airfoil performance to the very far wake," Wind Energy Science, Vol. 2, No. 1, jun 2017, pp. 317-328.

${ }^{34}$ Tan, J., Sun, Y., and Barakos, G., "Unsteady loads for coaxial rotors in forward flight computed using a vortex particle method," Aeronautical Journal, Vol. 122, No. 1251, 2018, pp. 693-714.

${ }^{35}$ Tan, J. F., Zhou, T. Y., Sun, Y. M., and Barakos, G. N., "Numerical investigation of the aerodynamic interaction between a tiltrotor and a tandem rotor during shipboard operations," Aerospace Science and Technology, Vol. 87, 2019, pp. 62-72.

${ }^{36}$ Jo, Y., Jardin, T., Gojon, R., Jacob, M. C., and Moschetta, J.-M., "Prediction of Noise from Low Reynolds Number Rotors with Different Number of Blades using a Non-Linear Vortex Lattice Method," 25th AIAA/CEAS Aeroacoustics Conference, , No. May, 2019, pp. 1-13.

${ }^{37}$ Yokota, R., Barba, L. A., Narumi, T., and Yasuoka, K., "Petascale turbulence simulation using a highly parallel fast multipole method on GPUs," Computer Physics Communications, Vol. 184, No. 3, 2013, pp. 445-455.

${ }^{38}$ Yokota, R. and Barba, L. A., "FMM-based vortex method for simulation of isotropic turbulence on GPUs , compared with a spectral method," Computers and Fluids, Vol. 80, 2013, pp. 17-27.

${ }^{39} \mathrm{Hu}$, Q., Gumerov, N. A., Yokota, R., Barba, L., and Duraiswami, R., "Scalable Fast Multipole Accelerated Vortex Methods," 2014 IEEE International Parallel 63 Distributed Processing Symposium Workshops, IEEE, may 2014, pp. 966-975.

${ }^{40}$ Greengard, L. F., The Rapid Evaluation Of Potential Fields In Particle Systems, Phd thesis, Yale University, 1987.

${ }^{41}$ Cheng, H., Greengard, L., and Rokhlin, V., "A Fast Adaptive Multipole Algorithm in Three Dimensions," Journal of Computational Physics, Vol. 155, No. 2, 1999, pp. 468-498.

${ }^{42}$ Yokota, R. and Barba, L. A., "Treecode and fast multipole method for N-body simulation with CUDA," GPU Computing Gems Emerald Edition, 2011, pp. 113-132.

${ }^{43}$ Yokota, R. and Barba, L. A., "Comparing the treecode with FMM on GPUs for vortex particle simulations of a leapfrogging vortex ring," Computers and Fluids, Vol. 45, No. 1, 2011, pp. 155-161.

${ }^{44}$ Rossi, L. F., "Resurrecting Core Spreading Vortex Methods: A New Scheme that is Both Deterministic and Convergent," SIAM Journal on Scientific Computing, Vol. 17, No. 2, mar 1996, pp. 370-397.

${ }^{45}$ Barba, L. a., "Vortex Method for computing high-Reynolds number Flows: Increased accuracy with a fully mesh-less formulation," California Institute of Technology, Vol. 2004, 2004.

${ }^{46}$ Barba, L., Leonard, A., and Allen, C., "Numerical investigations on the accuracy of the vortex method with and without remeshing," 16th AIAA Computational Fluid Dynamics Conference, , No. May 2014, 2003.

${ }^{47}$ Barba, L. A., Leonard, A., and Allen, C. B., "Vortex method with meshless spatial adaption for accurate simulation of viscous, unsteady vortical flows," International Journal for Numerical Methods in Fluids, Vol. 47, No. 8-9, 2005, pp. 841-848.

${ }^{48}$ Torres, C. E. and Barba, L. A., "Fast radial basis function interpolation with Gaussians by localization and iteration," Journal of Computational Physics, Vol. 228, No. 14, 2009, pp. 4976-4999. 
${ }^{49} \mathrm{Du}, \mathrm{Z}$. and Selig, M., "A 3-D stall-delay model for horizontal axis wind turbine performance prediction," 1998 ASME Wind Energy Symposium, American Institute of Aeronautics and Astronautics, Reston, Virigina, jan 1998.

${ }^{50}$ Viterna, L. A. and Janetzke, D. C., "Theoretical and experimental power from large horizontal-axis wind turbines," Tech. rep., Washington Procurement Operations Office, Washington, DC (United States), sep 1982.

${ }^{51}$ Brandt, J. B., Deters, R. W., Ananda, G. K., and Selig, M. S., "UIUC Propeller Database," http://mselig.ae.illinois.edu/props/propDB.html.

${ }^{52}$ McCrink, M. H. and Gregory, J. W., "Blade Element Momentum Modeling of Low-Reynolds Electric Propulsion Systems," Journal of Aircraft, Vol. 54, No. 1, 2017, pp. 163-176. 